\title{
The Performance of Knowledge Collaboration in Virtual Teams: An Empirical Study
}

\author{
Shuli Gao, Yanli Guo and Jianbin Chen \\ Business College of Beijing Union University, Beijing, China, 100025 \\ yanli.guo@buu.edu.cn
}

\begin{abstract}
Knowledge collaboration is an effective management method to promote the appreciations of intellectual capital and social capital. In a virtual team, team atmosphere, team network and the individual attributes, etc. shall affect the performance of knowledge collaboration (KC) in different degrees. The paper will baild the theoretical framework that affects the performance of virtual team knowledge collaboration, and use the Bootstrap-based structural equation model to perform empirical study on the theoretical framework. Empirical results show that network characteristics of virtual team, team attributes, individual attributes and collaborative efficiency all have significant impact on the performance of KC. Empirical conclusions will provide a theoretical basis and practical reference for promoting the performance of knowledge collaboration.
\end{abstract}

Keywords: Virtual Teams; Khorledge Collaboration; Performance

\section{Introduction}

With the rapid development and extensive application of information and communication technology, the fvirtual team collaborated across time, space and organizational boundary has beeome an important organizational structure for enterprises to cope with the increasingly accelerated market change. The virtual team, with advantages such as including diverse staff, broad organizational boundaries, flexible organizational structure and innovation resources allocation, etc., has been widely adopted in many fields ineluding service outsourcing, IT and innovation research and development, etc. The previous research has shown that a virtual team is an effective model to settle the complex task on wide-scope and cross-disciplinary knowledge demand within the organization [1]. Therefore, how to effectively improve the performance of virtual team has been a focused issue in the business and the academic circles.

From the perspective of knowledge management, the virtual team is essentially the use of "Heterogeneous" subjects for collaborative knowledge innovation. In addition to compensate for the knowledge gaps to effectively solve the problem of knowledge scenario embedding and path dependence, the knowledge collaboration model also can obtain collaborative effects of multi-subject, multi-target and multitasks. However, because of staff diversity, and the use of electronic information technology as the primary means of communication, lacking of face to face communication, the knowledge collaboration and performance of a team is facing many problems. Therefore, in terms of the characteristics of virtual teams, it is necessary to study how to further enhance the collaborative efficiency of the virtual team and improve the performance of knowledge collaboration by integrating the collaborative innovation resources. On the basis of defining the performance of knowledge collaboration and collaborative efficiency, and taking Chinese service outsourcing enterprises team as research objects, this paper performs empirical analysis on the critical factors influencing the performance of the virtual team's 
knowledge collaboration, and proposes corresponding suggestions on this research basis.

\section{Research Summary}

\subsection{Virtual Teams and Knowledge Collaboration}

\section{(1) Virtual teams}

Currently, there is no an agreed definition of virtual team. Lipnack and Stamps (1997) considered a virtual team as a group of people, inter-space, inter-time and interorganizational boundaries, who work independently from each other for a common target relying on the technology [2]. Kirkman et al., (2002) insisted that virtual teams were composed of employees with unique skills, located at a distance from each pher, who must collaborate to accomplish important organizational tasks, and put forward five challenges to virtual team success [3]. Zigrus (2003) summarized the concept of virtual team in the current research field preferably, he thought that virtual tean is a collection of individuals, and those individuals have the dispersion characteristics in different dimensions; the dispersion dimension mainly includes regional dimension, organizational dimension, time dimension and culture dimension, the individual works collaboratively via information and communication technologies, so as to achieve a certain goal [4]. Chen Yali (2009) holds that, in terms of the establishment of a virtual team, the team member mainly consists of scientific and techinical personnel based on knowledge, skills and capabilities, and pays more attention to individaal capabilities, cooperative capability, and the matching degree of adaptation, etc., [5]. ER Yanyong (2013) thought that the fields adopting virtual team as the organizational form are mainly concentrated in the innovative field, involving in fields such as high and new technology, design and research and development, software development scientific research and consultation, etc., and its knowledge or product is the main output [6]. Therefore, for the virtual team, from the perspective of either the team's fornation of target and output of purpose, or the team's own characteristics, the knowledge innovation is the soul of its existence.

\section{(2) Knowledge collaboration}

Karlenzig (2002) first put forward the concept of "Knowledge Collaboration", he thought that it is an organizational strategy, which could dynamically assemble the internal and external systems, business process, technology and relation (community, customer, parther and the supplier), to maximize the business performance[7]. After this, many seholars proposed different definitions from different perspectives and levels. From the enterprise perspective, Mckelvey et al., (2003) thought that knowledge collaboration is a kind of "Activity", such as, collaborative development and collaborative authoring, etc.; the members participated in the activity strive to create personal knowledge and form a valuable result eventually [8]. Tong Zehua (2012) comprehensively thought knowledge collaboration is a kind of effective collaborative status in time and space achieved by the subject, object and environment, etc. in the knowledge management, and achieves at the right time and space, and it is multidimensional dynamic process that transferring appropriate information and knowledge to appropriate target or object to achieve knowledge innovation. Knowledge collaboration is the advanced stage of knowledge management, and its ultimate target is to achieve knowledge innovation, which is the target of virtual teams [9]. In addition, at the micro level, Leijen and Baets (2003) pointed out that knowledge collaboration refers to a knowledge requester realized that himself/herself does not have the capacity to solve a problem, while another knowledge provider just having the capacity in this aspect, if both parties could reach a consensus, 
they can integrate their knowledge to make for the knowledge demand of the knowledge requester, so as to solve the problem [10]. At the macro level, Chen Kunyu and Chen Kunqiong (2002) thought that via integrating the internal and external knowledge resources of the organization, the enterprise could make the overall efficiency of organizational learning and knowledge utilization and creation greater than the sum of each independent component, and its purpose is to obtain the knowledge collaborative effect of " $1+1>2$ " [11]. Since the micro individual can solve problems through knowledge collaboration, and the macro-organizations can create benefits through knowledge collaboration as well, it is a necessary action to research the performance of knowledge collaboration.

\subsection{Knowledge Collaboration Performance Model}

The so-called "Performance" refers to the final results showing the overall operational activities of the organization, meanwhile, it is a factor to measure the degree of saccessful achievement of the organization's tasks or targets [12]. In performance of knowledge collaboration, the current researches are mainly focused on the effect of /or the relation between the knowledge management, knowledge sharng, knowledge transfer and knowledge collaboration, etc. on organizationa performanet enterprise performance, team performance, management performance and innovative performance, etc. For example, Wan Youqing and Deng Mingran (2007) analyzed the collaborative innovation performance of industry cluster based on the angle of knowledge view, and constructed the collaborative innovation performanee model of industry cluster from the aspects of enterprise knowledge's base stock, enterprise innovation power, innovation capacity and the collaboration between enterprises, etc., [134. Du Baocang and Li Chaoming (2009) built a relevant indicator system for the cellaborative work performance of the interorganizational knowledge workers, and cartiled out an application example using the multi-level extension evaluation method [14]. Xu Qiang and Jian Zhaoquan et al., (2010) constructed the modêl on the mutual relation between network relation, trust, knowledge sharing and the technology innovation performance, through empirical research, they believed that: the degree of rust, network relation and knowledge sharing between enterprises have positive influence on the enterprise's technology innovation performance [15]. Zheng Xlaodan (2010) started with the organizational collaboration of parentsubsidiary Corporation, laking knowledge collaboration as intermediary variable, analyzed the relation among the organizational collaboration, knowledge collaboration and innovation peformance of the parent-subsidiary corporation, and constructed corresponding conceptual model [16]. Zhou Fang and Guo Yan (2012) built a theoretical model of tripartite relation of supply chain social capital, knowledge sharing and innovation performance, and holds that, through empirical research: There is a positive relation between social capital and supply chain enterprise innovation performance, knowledge sharing between supply chain enterprises plays an important intermediary role in social capital and supply chain enterprise innovation performance [17]. By taking knowledge integration as intermediary variables, Shi Liping et al., (2013) studied the mechanism of interaction memory systems and knowledge team performance. In this literature, they constructed interaction memory system, the theoretical framework of the relation between knowledge integration and performance of knowledge group, but the measurement of performance of knowledge group did not be subdivided. Nevertheless, this literature proves that knowledge integration between internal and external knowledge has a positive impact on performance of knowledge group; this conclusion explains the necessity and feasibility of the performance of knowledge collaboration in virtual teams' research from a side [18].

From the existing literature, empirical research on the measurement and influencing factors of performance of knowledge collaboration is insufficient. For existing literatures 
discussing the performance issues of knowledge from the knowledge itself, it only stay in discussing the performance of knowledge sharing and effect of knowledge transfer and did not mention the performance, result or efficiency measurement of knowledge collaboration. In fact, knowledge collaboration is a complex process including knowledge sharing, knowledge transfer and knowledge creation [19].

\subsection{Knowledge Management based on Social Network}

Research on knowledge management and knowledge transfer based on social network perspective is not a new topic. As early as 1999, Seufert considered knowledge network is a kind of social network among knowledge participants to achieve value creation through knowledge creation and transfer in levels such as individuals, groups, organizations and interior, etc., [20]. Hansen (1999) presented an opinion that weak ties is more conducive to knowledge transfer, opening a prologue of researching on knowledge spraring and knowledge transfer from the perspective of social networks and social relation[21]. Singh (2005) researched the knowledge collaboration network, $\{$ and provided a knowledge network collaborative model. Currently a lot of research literatures adopt empirical research forms to argue in different levels, which am at different social networking characteristic' influence relation to knowledge management, knowledge sharing or knowledge transfer [22]. For example, Inkpen etal., (2005) used the social network method to construct the theoretical framework of knowledge transfer among virtual teams, to research how to organize the organizational knowledge of virtual team and the incentive problem of how to solve the problem of task knøwledge, they found that social relations including trust, and emotionaijoint, etc. make knowledge transfer easier [23]. Stephen and Irene (2008) researched how to apply social network-based system to support interactive collaboration in knowledge sharing over peer-to-peer networks [24]. Zhu (2009) demonstrated the influence of the hetwork characteristics (associative strength, network density, network seope and nethork centrality) of knowledge resource enterprise and knowledge receiving enterprise on the knowledge transfer effect among enterprises, by taking the knowledge transfer effect as research object, and taking the individual attributes of knowledge resolarce enterprise and knowledge receiving enterprise as intermediary variable [25]. Yanli (2010) took a particular virtual team as example, used social network method to andlyze the knowledge sharing mechanism within the virtual team; the research found that the emotional joint among the team members is beneficial to knowledge sharing and the network accessibility and the number of small group will influence the efficrency of knowledge sharing within the team [26]. Shen Huimin (2013) researched the impact of social network characteristic on community knowledge creation. She considered that community network characteristics (network density and connection strength) haye important influence on community knowledge creation. Good community networks are conducive to the formation of effective collaboration atmosphere; and high connegtion strength among community members is conductive to increase collaborative work opportunities to improve the performance of social knowledge creation [27].

The above existing research shows that the function that social networking characteristics on knowledge management have been demonstrated, the existence of influence relation is positive. The difference is that under different research objects and different research situation, the play mechanism may be different; and knowledge collaboration is a dynamic process, and its play mechanism will be different as well. Therefore, in the study of performance of knowledge collaboration in virtual teams, social networking characteristic will be an indispensable key factor.

\subsection{Critical Review}

If we take the virtual team as a system, we could decide, no matter from the input (the composition of the team and resource access) or from the output (form of output: new 
products and new knowledge), that the virtual team is existed for the purpose of knowledge innovation. And effective knowledge collaboration mechanism is a good way for knowledge creation and knowledge innovation. Although there are many literatures discussing the problems related to knowledge management of virtual teams, but currently research on performance of knowledge collaboration in virtual teams is insufficient. Knowledge collaboration is a dynamic process, throughout the process of knowledge transfer, knowledge sharing, and knowledge innovation. Although it may contain links or stages such as knowledge sharing, knowledge transfer, knowledge learning and knowledge creation, etc., the performance of any single link is unable to represent the overall performance of knowledge collaboration. In consequence, this research will focus on the measurement of the performance of virtual team's knowledge collaboration, and analyze the principal factors influencing the performance of the virtual team's knowledge collaboration from the social network perspective, the attribute of virtual team the individual attributes and the efficiency of knowledge collaboration, etc.

\section{Theoretical Models and Assumptions}

\subsection{Performance of Knowledge Collaboration (KC) and its Measurement}

Knowledge collaboration $(\mathrm{KC})$ is the process of interpersonal Knowledge interaction; knowledge subjects integrate their own knowedge with that gatned from others to form new knowledge. Due to the intangibility, Sharing competency and non-depreciation of knowledge capital, in the effectiveness or $\mathrm{KC}$, except for the final intellectual products (taking products or service as carfier) deliverng to the customer, the intellectual achievements contained in the interlectual products also become the appreciation part of enterprise's knowledge capital which are refleeted as the capability improvement of individual and the team, the accumulation of experience and the improvement of process, and also reflected as the increase of explicit knowledge (the increase of experience summary, process doctmentation and knowledge base, etc.). In addition, knowledge collaboration is the process in Jwhich individual knowledge demanders look for knowledge providers and exchange their knowledge. In this process, the connection among subjects of knowledge tend to be more and firmer; the trust, reciprocity and mutual respect approval among subjects tend to be enhanced; their languages, stands and viewpoints tend to be uhanimous. These increments formed the increase in social capital amount. Therefore performance of knowledge collaboration can be measured from appreciation of tro aspects including the knowledge capital and social capital.

(1) Measurfement of knowledge capital

Knowledge capital includes all the assets created by intellectual activities including knowledge acquisition, innovation and establishment of value chain, etc. Knowledge collaboration is the process of knowledge creation, in the process, the capabilities of knowledge worker are promoted, the knowledge gets renewals and extensions and the organization environment and business process get improved, all these belong to the category of knowledge capital (Chen, 2014)[28]. Therefore, the results of knowledge collaboration will lead to the increase of knowledge capital; in other words, the appreciation of knowledge capital can be used to measure the effectiveness of knowledge collaboration.

(2) Measurement of knowledge capital

From the perspective of epistemology, knowledge creation has three modes, namely, cognitivists, autopoietics and connectionists; it is obvious that connectionists is consistent with the knowledge creation of knowledge collaboration, which could be explained as that the intellectual individual triggers the connection between each other in solving the 
collaborative problem, and the new connection results in new knowledge. The connectionists introduced the concept of social capital to knowledge collaboration, considering that social capital is the interest, value, or assets, etc. generated by social connections and social interactions. In the analysis of knowledge creation, numerous researchers take social capital as one of the major researching elements (Nahapiet \& Ghoshal, 1998) [29].

The purpose of knowledge collaboration is knowledge creation. The knowledge creation of virtual teams is the process of constantly acquiring social capital through social network. The social capital of virtual team has a positive role in promoting knowledge innovation, which is advantageous to the knowledge collaboration; knowledge collaboration means that the reinforcement of the connection in knowledge network and the increase of new connection, promoting the appreciation of social capital (Chen, 2014) [28]. Therefore, good knowledge collaboration will lead to the increase of social capital; in other words, the appreciation of social capital can be used to measure the effectiveness of knowledge collaboration.

\subsection{Social Network Characteristics and Performance of KC}

(1) Network density

Network density is the structural index of social network, which refers to the ratio of numbers of actual presence connections and possible confrections in the network (Wang, 2009)[30]. It describes the close degree between network nodes. The higher density network is, the more closely node links. When the hetwork density approaches to the value of 1 , there are strong direct links between one and each other node, which means every node is in the "eye sight" of any other node. McFadyen (2004) believes that the node relations in the network structure are conducive to form the strong trust mechanism, which has the positive function to form this trust mechanism for knowledge innovation and cooperation relations $[31]$.

In virtual teams each node is an independent knowledge carrier, among these individuals there is a network structure of knowledge circulation established in a flexible manner across time and space. In the network virtual team, the higher the network density is, the closer the relation anong knowledge carriers are, and the more internal knowledge sharing nodes are, and the better the effect of knowledge collaboration is(Wang, 2009)[30]. Network density has important influence on the transfer effect of individual knowledge (Fritsch er al., 2010) [32].The higher the network density is, the closer the relationship between individuals of virtual team is, and the more easy to form the trusted channel of knowledge transferring, and also more helps to improving performance of KC. Therefore, we put forward the following hypothesis:

H1a Network density has positive influence on performance of $\mathrm{KC}$; the higher the netrork density is, the higher performance of $\mathrm{KC}$ is.

\section{(2) Network centrality}

"Centrality" is the basic concept of distinguishing network status in social network analysis, which is used to measure which level the individual or organization is positioned in social networks. "Point of potential" and "degree centrality" is the two kinds of index. The former is used to describe the overall centrality of network graph, and the latter is used to describe the centrality of any single point in a network. This paper will use the "Point of potential" to describe network centrality.

According to social network theory, "Point centrality" is measured by the number of points in the network which directly connect to it. If an individual point is directly connected to many other points, then it means the point has high degree "Point 
centrality". In the structure of a network, a node, which is directly connected to other nodes, is the central position of network structure, and has great power of resource acquisition.

Social network provides effective knowledge circulation and collaborative innovation platform for knowledge collaboration. The key node in the central position of a network plays an important role in the network structure collaboration and knowledge sharing. Chen Lu et al., (2009) stated that individual relation network variable such as network structure (centrality) and the diversity of knowledge has positive influence on the virtual team's performance [33].Reinholt et al., (2011) noted that network centrality would foster network positions that provide access to nonredundant knowledge [34]. According to social network theory, if the individual has higher degree of centrality in the network, then he or she has more contact with other individuals, and there is more channei for him or her to exchange knowledge with other individuals, and therefor promote the knowledge collaboration. Thus, in virtual teams of social networks, the higher degree ndividual centrality are, the higher performance of $\mathrm{KC}$ is. Therefore, we put foryard the following hypothesis:

H1b: Network centrality has positive influence on performance of $\mathrm{KC}$, the higher degree of network centrality is, the higher performance of $\mathrm{KC}$ is.

(3) Connection strength

The network density and centrality is the structure dimension index of social network, while the connection strength is relational dimension/index. The relationship is a beneficial supplement to the dimensions of the structures (Yao \& Ming, 2008) [35]. There are many researches about the infleence of the connection strength on knowledge sharing and knowledge transferring. The study shows the connection strength plays a very important role in the process of knowledge transferring among the groups (Hansen, 1999; Reagans \& McEvily,2003, Uzzi \& Lâneaster, 2003; Zhaoguo, et al., 2011) [21,36-38]. The shorter and the more number tbe individuals connected, the more knowledge acquisition node in the team, the shorter the project completion time is. In the same meaning, the higher connecting strength is, the higher the accuracy and timeliness of knowledge collaboration is. Therefore, we put forward the following hypothesis:

$\mathrm{H} 1 \mathrm{c}$ : Connection strength has positive influence on performance of $\mathrm{KC}$.

In summary, we can assume that:

H1: The characteristics of social network and the performance of $\mathrm{KC}$ is positive correlation. To strengthen the social network construction is conducive to enhancing the performance of KC.

\subsection{Individual Attributes and Performance of $\mathrm{KC}$}

(1) Collaborative intention

Knowledge collaboration is usually used for completing a knowledge-intensive task, therefore, the team members should have strong collaborative intention, and operate various forms of knowledge capital in the collaborative process, and complement knowledge to each other, which is the important characteristic of knowledge collaboration, and it also the basis to perform collaboration among the knowledge subjects, especially that knowledge is implicit, which is required the team members having strong collaborative intention. Jente (2008) investigated 499 respondents and found that individual attitudes had significantly impact on organizational knowledge sharing[39]. In the research of this paper, collaborative intention refers to the subjective desire of whether the team members and to what extent are willing to share their own knowledge to other members, and are willing to accept the knowledge of other members, which is a two-way process. Knowledge collaboration not only can contribute to the 
transformation and upgrading of individual knowledge, but also can support team members to achieve their own work targets. In the team collaboration, the team member often comes from different professional fields and plays different roles, only on the premise that the employees approve the concept of knowledge collaboration, can they make a positive contribution of their own knowledge and constantly absorb knowledge to achieve knowledge innovation. Therefore, the individual collaborative intention will exert great influence on the energy and time, etc., devoted into the knowledge sharing, knowledge transfer, organizational learning and knowledge creation by the team members, so as to influence the performance of knowledge collaboration correspondingly. Therefore, the following assumption is put forward:

$\mathrm{H} 2 \mathrm{a}$ : Individual collaborative intention has positive influence on the promotion of performance of $\mathrm{KC}$.

(2) Collaborative capacity

In addition to the collaborative intention, another important factor affecting the employees' knowledge collaboration behaviors is employees' indifidual collaborative capacity. The individual collaborative capacity includes not only the capability to assess the own needs of the individual accurately, and nore importantly it includes the capacity of whether the individual could clearly express to other mémbers their own knowledge and the capability to absorb knowledge. These collaborative capabilities of the team member may partly be the result of their Oyn talents, but mostly they are affected by postnatal environment, such as organizational training, erc. When team members have good collaborative capacities, the transformed quanlity, quality, speed and effectiveness of the knowledge will be greatly improved and the promotion of performance of knowledge collaboration will be more remarkable. Therefore, the following assumption is put forward:

$\mathrm{H} 2 \mathrm{~b}$ : Individual collaborative capacties have positive influence on the promotion of performance of $\mathrm{KC}$.

In summary, we can assume thatt:

$\mathrm{H}$ 2: Individual attributes and petformance of $\mathrm{KC}$ are positive correlation and strong intention of individual collaboration and collaborative capacity will facilitate improvement of performance of KC.

\subsection{Team attributes and performance of $\mathrm{KC}$}

(1) Collaborative atmosphere

Sveiby and Simons (2002) conducted in-depth research on the collaborative atmosphere in their paper, they thought that collaborative atmosphere refers to the value, belief and imagine, etc. influencing knowledge sharing and the knowledge creation behavior and intentions, and they conducted measurement from four aspects of employee attitude, work group support, direct manager and the organizational culture [40]. In the knowledge collaborative process, the knowledge innovation is accelerated to be achieved through vast collaborative effect by team members with different knowledge background, and the collaboration among members is nothing else than the formative effect of collaborative atmosphere. In good collaborative atmosphere with information sharing, mutual respect and mutual trust, team members with different perspectives and ways of thinking are more willing to share own talent and experience inside the and among organizations. Communication and cooperation among members will facilitate the search, transfer and creation of knowledge and increase intellectual capital, social capital, ultimately improving the performance of $\mathrm{KC}$. Therefore, the following assumption is put forward: 
$\mathrm{H} 3 \mathrm{a}$ : Collaborative atmosphere and performance of $\mathrm{KC}$ are positive correlation and good collaborative atmosphere will facilitate improvement of performance of $\mathrm{KC}$.

(2) Collaborative culture

Collaborative culture refers to the beliefs, value system and behavioral norms formed in the organizational learning process of the team and obtained the mutual recognition from the team members, which is mainly reflected in aspects including cohesion, core concept and team spirit, etc. Collaborative culture is a kind of intangible assets, it is difficult to describe and imitate its value, and it is formed through constantly breaking-in and accumulation in the long-term practice process of the team members, which has binding effect on the behavior and consciousness of the team members. Under the common value theory and core concept, the members will enjoy freedom, independenee and being respected at work, and will form a strong sense of belonging to the tean, the members will devote themselves to the team work with the co-sensed "Tedm 'Spirit" comprehensively, to create amazing achievement. Previous researches such as López et al (2004) and Li, et al., (2009), found that collaborative cuttyre has a significant positive correlation to organizational performance and knowledge shating through empirical research [41-42]. We believes that relaxed and hanmonious, colearning and intermotivated collaborative culture will help the team members to exchange knowledge and share resources and produce new ideas and thoughts, and then promoting the performance of KC. Therefore, the following assumption is put forwård

$\mathrm{H} 3 \mathrm{~b}$ : Collaborative culture and performance of $\mathrm{KC}$ are positive correlation and good collaborative culture will facilitate improvement of performance of KC.

\section{(3) Motivator}

Motivation is a process that can stimufate people's behavioral motive to generate a specific behavior. In the team collaboration process, motivation measures is an effective mean of team management, which could not only promote the knowledge capability of the organization and the members, but can empower the organization and the member with sustainable power and core competition advantages. In the knowledge collaboration process, need to build scientific and effective motivation mechanism based on individual level and team level to Anprove the performance of knowledge collaboration. By giving the affirmation of both spiritual and material to the contribution of the team members promptly, and obtaming abundant financial support in the work activities, could not only provide opportunity, for the members to express and develop themselves, but could attract and encourage exchanging, sharing, integrating and creating knowledge to each other, to realize knowledge innovation finally. Therefore, the following assumption is put forward:

$\mathrm{H} 3$ c.Motivator and performance of $\mathrm{KC}$ are positive correlation and good motivation system will facilitate improvement of performance of $\mathrm{KC}$.

In summary, we can assume that:

H3: Team attributes and performance of $\mathrm{KC}$ are positive correlation, and good collaborative atmosphere, cultural and effective motivator, etc., will facilitate improvement of performance of $\mathrm{KC}$.

Based on the above analysis, the theoretical model of this paper is shown in Figure 1: 


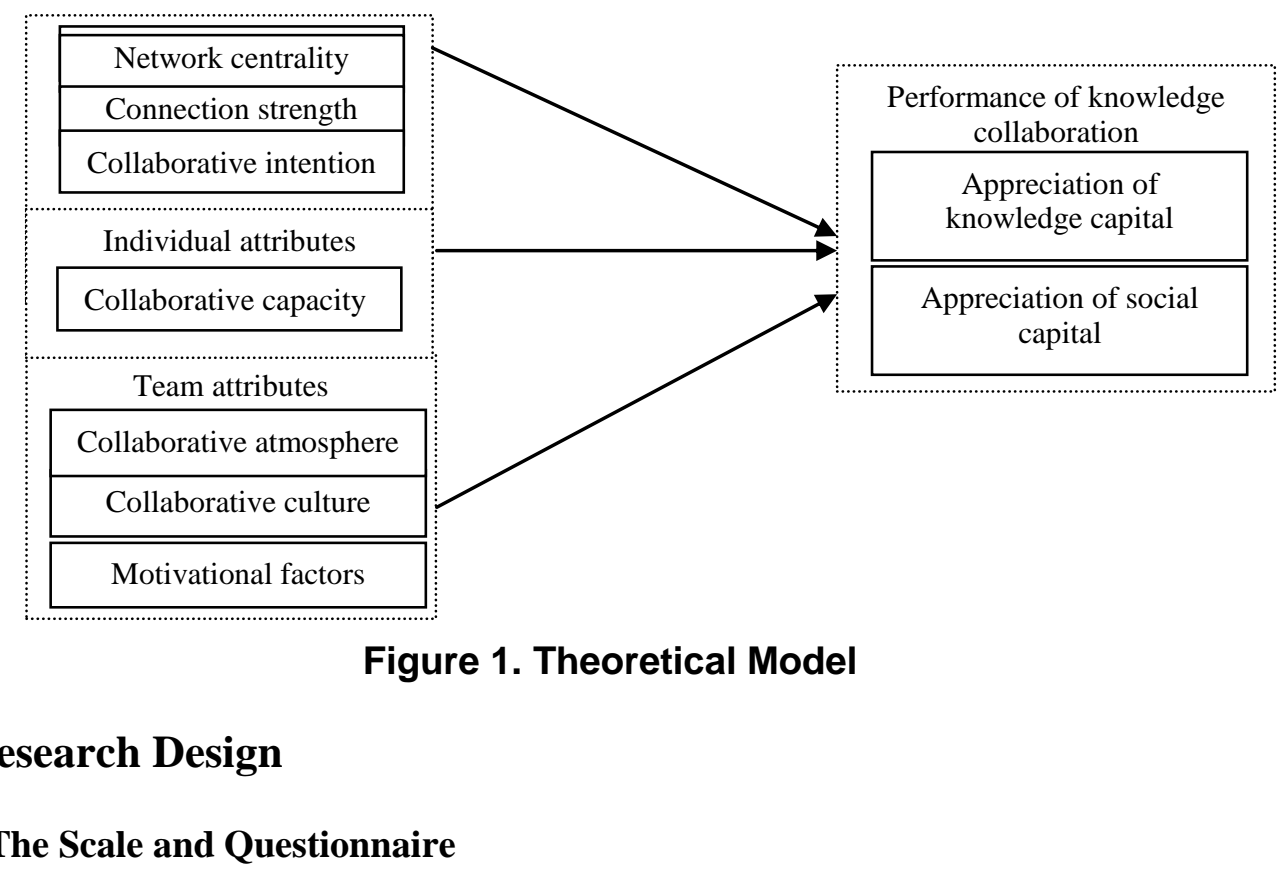

\subsection{The Scale and Questionnaire}

This paper uses structural questionnaire to gather dâta, and the measurement index in the paper mainly uses the existing research scale at domestic and overseas, and combines with the knowledge collaboration characteristics of Chinese service outsourcing enterprises to improve the observational varjables, to further adjust and amend the questionnaire items via interviews and pre-exmination, and the final questionnaire includes 35 items. Among which, there are Pespectively three items for network density and network centrality; there are 5 -items for network connection strength; there are respectively 5 items for team collaboration atmosphere and collaboration culture; there are 4 items for motivator; there are respectively 5 items for the two dimensions of performance of KC, namely the appreciation of knowledge capital and social capital. Scoring formula of questionnaire adopts Likert scale with 5 points, in which "1" representing strong disagreenent and "5" representing strong agreement. Measurement dimension and summary of index of each variable are shown in Table 1.

\section{Table 1. Variables Measuring Dimensions}

\begin{tabular}{|c|c|c|c|}
\hline Variables & $\begin{array}{l}\text { Measuring } \\
\text { dimension }\end{array}$ & Meaning of index & $\begin{array}{c}\text { References of } \\
\text { measuring items }\end{array}$ \\
\hline \multirow{3}{*}{$\begin{array}{l}\text { Network } \\
\text { Characteristics }\end{array}$} & Network density & $\begin{array}{lcc}\text { Connected } & \text { number } & \text { and } \\
\text { tightness between nodes } & \\
\end{array}$ & \multirow[t]{2}{*}{$\begin{array}{l}\text { Luo, 2009[43]; } \\
\text { Zhu, 2009[44] }\end{array}$} \\
\hline & $\begin{array}{l}\text { Network } \\
\text { centrality }\end{array}$ & $\begin{array}{l}\text { Amount of direct contact } \\
\text { between other nodes and the } \\
\text { some node in the consulting } \\
\text { network; understanding the } \\
\text { level and consulting intention }\end{array}$ & \\
\hline & $\begin{array}{l}\text { Connection } \\
\text { strength }\end{array}$ & $\begin{array}{l}\text { Recognizing duration, interact } \\
\text { frequency, intimate topics and } \\
\text { intimate action }\end{array}$ & $\begin{array}{c}\text { Granovetter,1973[45]; } \\
\text { Masden\& } \\
\text { Campbell,1984[46] }\end{array}$ \\
\hline \multirow{2}{*}{$\begin{array}{l}\text { Individual } \\
\text { Attributes }\end{array}$} & $\begin{array}{l}\text { Collaborative } \\
\text { intention }\end{array}$ & Initiative and enthusiasm & Zhu, 2009[44]; \\
\hline & $\begin{array}{l}\text { Collaborative } \\
\text { capacity }\end{array}$ & $\begin{array}{l}\text { Usage of tools and hold of } \\
\text { collaboration processes, etc. }\end{array}$ & Zhui, 2009[44] \\
\hline
\end{tabular}




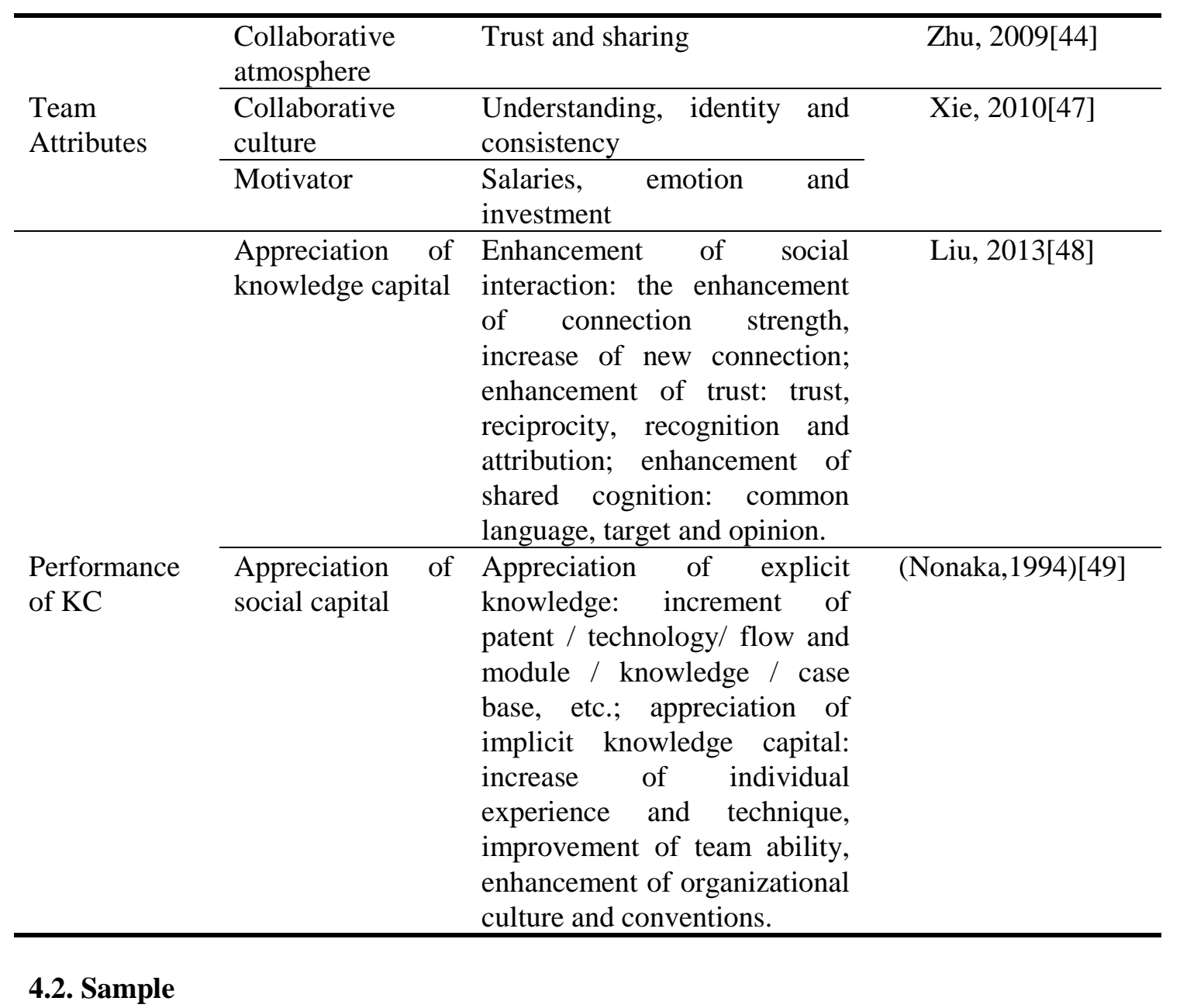

This research takes the service outsourcing enterprise team as the research subject, entrusts the third party research company to carry out a questionnaire inquiry for two months from October to December, 2013. There are 266 copies of questionnaires being recovered, of which 235 copies are valid, with the effective recovery rate of $88.3 \%$. In this survey, the projecteams from Beijing Baidu Network Technology Co., Ltd., Beijing Topsec Software Co., Ltd., Glodon Software Company Limited, Beijing Tongfang Dingxin Information Technology Co., Ltd. and Dongfang Futong (Beijing) Software Co., Ltd., etc., a total of 27 enterprises received the survey. These teams are the virtual teams in the Research and Development and innovation field, and the positions of the respondents in the team include Research and Development Manager, Project Manager, Senior Consultant, Engineering and developer, etc.; they have better familiarity and sensitivity on the items involved in the questionnaire, regardless of their work experience, expertise or qualifications, and the respondents have higher pertinence.

\section{Data Analysis and Hypothesis Test}

\subsection{Reliability and validity test}

Reliability of variables or factors is tested with Cronbach's $\alpha$ coefficient in this research, and its results are shown in Table 2. Cronbach's $\alpha$ value of variables or factors are more than 0.7 , indicating that the scales used in this research have a good reliability. In terms of validity, this research has a good content validity to a certain extent since the questionnaire used in this research draws on the existing literature scales, and has been 
consulted, pre-tested and modified by experts and business people. To further examine the structure validity of scales used, the specific items are inspected by confirmatory factor analysis (CFA) in this paper, and its results are shown in Table 2. KMO values of network characteristics, individual attributes, team attributes and performance of $\mathrm{KC}$ are more than the minimum level of 0.5 , which are significant. Factor loading of each item is more than 0.5 , indicating that factors have a stronger explanatory power to the corresponding latent variables; therefore the questionnaire has good structure validity.

Table 2. Validity and Reliability

\begin{tabular}{|c|c|c|c|c|c|}
\hline Variable & \multicolumn{2}{|l|}{ Dimensions and Items } & Factor Loading & KMO & Cronbach's \\
\hline \multirow{3}{*}{$\begin{array}{l}\text { Network } \\
\text { characteristics }\end{array}$} & Network density & wlmd_1 wlmd3 & $0.565 \sim 0.683$ & \multirow{5}{*}{0.768} & \\
\hline & Network centrality & wlzxd_1 wlzxd_3 & $0.610 \sim 0.739$ & & 0.815 \\
\hline & Connection strength & ljqd_1 1jqd_5 & $0.591 \sim 0.778$ & & \\
\hline \multirow{2}{*}{$\begin{array}{l}\text { Individual } \\
\text { attributes }\end{array}$} & $\begin{array}{c}\text { Collaborative } \\
\text { intention }\end{array}$ & & & & \multirow{2}{*}{0.802} \\
\hline & $\begin{array}{c}\text { Collaborative } \\
\text { capacity }\end{array}$ & & 814 & & \\
\hline \multirow{3}{*}{$\begin{array}{l}\text { Team } \\
\text { attributes }\end{array}$} & $\begin{array}{l}\text { Collaborative } \\
\text { atmosphere }\end{array}$ & & $0.558 \sim 0.805$ & \multirow{3}{*}{0.838} & \multirow{3}{*}{0.841} \\
\hline & Collaborative culture & tdwh=1 $\sim \operatorname{tdwh} 5$ & $0.612 \sim 0.713$ & & \\
\hline & Motivator & tdj1_1 _taji (4) & $0.763 \sim 0.825$ & & \\
\hline \multirow{2}{*}{$\begin{array}{l}\text { Performance } \\
\text { KC }\end{array}$} & $\begin{array}{l}\text { Appreciation } / \text { of } \\
\text { knowledge capltal }\end{array}$ & zszb_1_zszb_5 & $0.671 \sim 0.828$ & \multirow{2}{*}{0.865} & \multirow{2}{*}{0.859} \\
\hline & $\begin{array}{c}\text { Appreciation of social } \\
\text { capital }\end{array}$ & shzb_I $\sim$ shzb_5 & $0.648 \sim 0.812$ & & \\
\hline
\end{tabular}

5.2. The impact of network charaeteristics, individual attributes and team attributes on performance of $\mathrm{KC}$

Let network density network centrality and connection strength as the independent variables, performaneg of knowledge collaboration as the dependent variable. Then we can use SPSS 19.0 software to do regression analysis. Similarly, regression analysis is performed through letting Individual collaborative intention, individual collaborative capacity as the independent variables, performance of $\mathrm{KC}$ as the dependent variable. We can also proceed regression analysis with collaborative atmosphere, collaborative culture, motivator and performance of KC. The results are shown in Table 3.

Table 3. The Impact of Network Characteristics, Individual Attributes and Team Attributes on Performance of KC

Hypothesis Assuming path
Standardized regression coefficients results

(significance level)

\begin{tabular}{llll}
\hline H1a & Network density $\rightarrow$ Performance of KC & $0.105^{* *}$ & Support \\
H1b & Network centrality $\rightarrow$ Performance of KC & $0.152^{* * *}$ & Support \\
H1c & Connection strength $\rightarrow$ Performance of KC & $0.197^{* * *}$ & Support \\
H2a & Collaborative intention $\rightarrow$ Performance of KC & $0.307^{* * *}$ & Support
\end{tabular}




\begin{tabular}{llll} 
H2b & Collaborative capacity $\rightarrow$ Performance of KC & $0.209^{* * *}$ & Support \\
H3a & $\begin{array}{l}\text { Collaborative atmosphere } \rightarrow \text { Performance of } \\
\text { KC }\end{array}$ & $0.235^{* * *}$ & Support \\
H3b & Collaborative culture $\rightarrow$ Performance of KC & $0.348^{* * *}$ & Support \\
H3c & Motivator $\rightarrow$ Performance of KC & $0.186^{* *}$ & Support \\
\hline
\end{tabular}

Note. $* * p_{-} .05, * * * p_{-} .01$.

By analyzing data, we found that network density, network centrality, the connection strength have positive influence on performance of KC. It means that the performance of $\mathrm{KC}$ will be better when network density, network centrality and connection strength are rising. In addition, the collaborative willingness and ability of individuals affectpositively on the performance of KC. Similarly, collaborative atmosphere, collaborative culture and motivator have positive influence on the performance of $\mathrm{KG} \mathrm{So}$, the assumptions of H1a, H1b, H1c, H2a, H2b, H3a, H3b, H3c are supported.

\subsection{Structural Equation Analysis}

We will using AMOS19.0 structural equation modeling(SEM) software to study the mechanism between variables. The path diagram is shown th Figure 2.

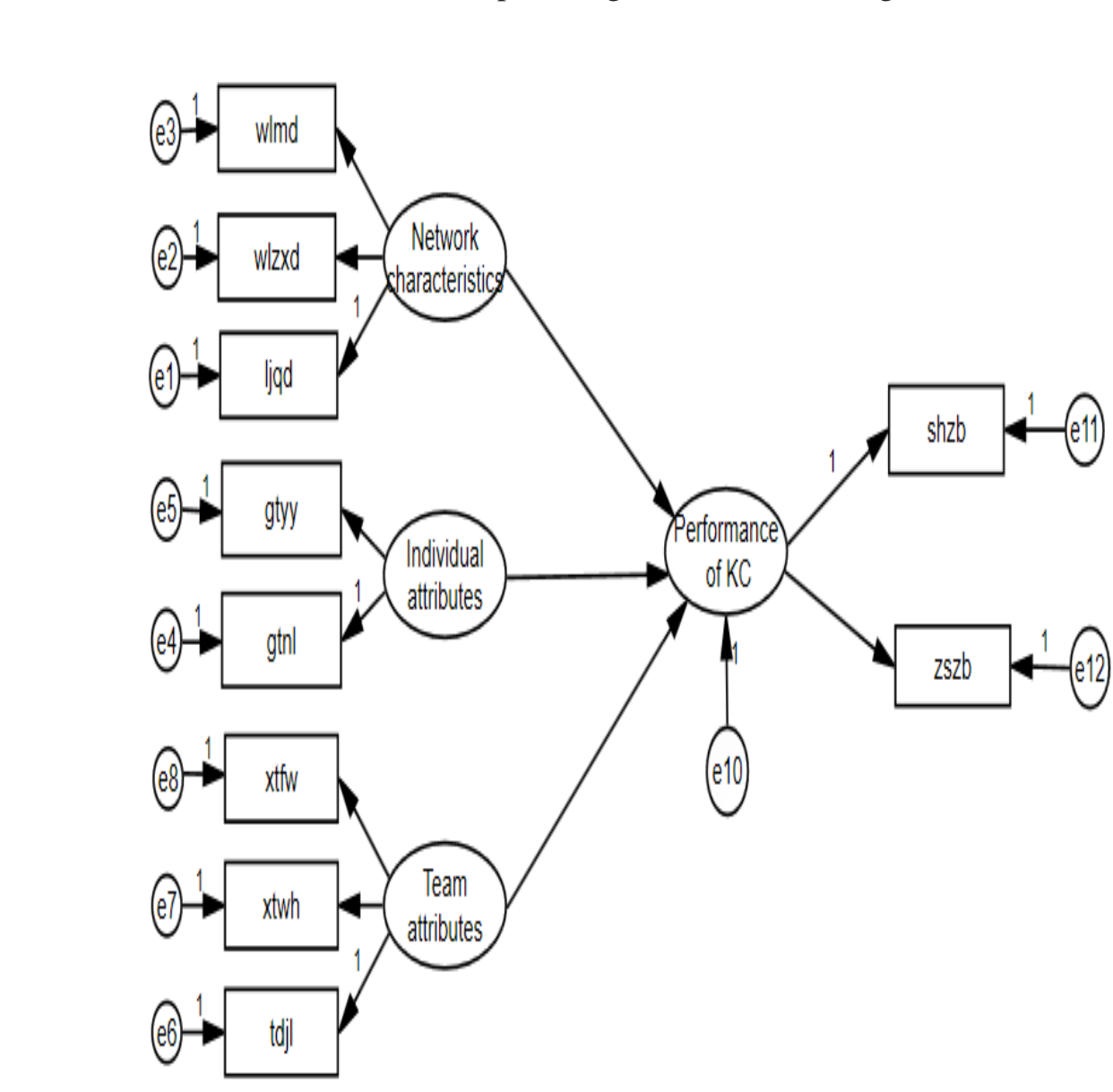

Figure 2. Path Diagram

Since SEM using the maximum likelihood method, this method requires that the data should be compliance with the assumption of multivariate normality, otherwise chi-square 
estimates will swell and the model fit will become deterioration. Therefore the data should be tested firstly whether the data meet the assumption of multivariate normality. The results are shown in Table 4.

\section{Table 4. Joint Normal Distribution Test Results}

\begin{tabular}{lll}
\hline Index & Kurtosis & Critical value \\
\hline Multivariate & 37.972 & 18.839 \\
\hline
\end{tabular}

Hau Kit-tai, etc., (2004) pointed out that when data's absolute value of kurtosis is less than 10, or critical value is less than 5 , it indicates that data follow the normal distribution[50]. Table 4 shows that mmultivariate kurtosis is equal to 37.972 and critical value is 18.839 . Both values exceed the threshold value. Therefore it can be considered that the data does not meet the assumption of multivariate normality.

Since the data is non-normal distribution, we use the Bootstrap method to do path path analysis and verify impact mechanism among network characteristics, individual attributes, team attributes and the performance of KC. In the AMOS tructural equation model, we set the 2000 Bootstrap samples in Bollenstine Bootstrap operation and achieved the convergence in 19 iterations. The results show that the model has a good fit in 1837 Bootstrap samples, and the poor fit in 163 Bootstrap samples. The significant probability of Bollen-Stine Bootstrap is 0.082. Thus, although the data does not meet the assumption of joint normal distribution, but the fitness of the model is acceptable.

The results of path analysis show that absolute fi andex $\chi^{2} / d f$ is equal to 3.27, more than 3, GFI is 0.877 , less than $0.9 \%$ RMSEA is 0.082 , more than 0.08 ; relative fit index NNFI is 0.903 , IFI is 0.832 CFI is 0.902 , these index do not all meet the above 0.9 requirements. Overall, the data Aailed to better fit the research model and the original model should be modified We can use the modified index (MI) provided by AMOS19.0 to adjust the theoretical models. By adding the error covariance in "wlmd wlzxd", "gtnl $\sim$ gtyy", "xtfw $\sim$ xt he, we can get the following results (see Table 5).

\begin{tabular}{|c|c|c|c|c|c|c|c|}
\hline Fit Index & $x=/ d f$ & $\overline{\mathrm{GHO}}$ & RMSEA & RMR & IFI & NNFI & $\overline{\mathrm{CFI}}$ \\
\hline Value & \begin{tabular}{|l|}
2.574 \\
\end{tabular} & 0.927 & 0.058 & \begin{tabular}{|l|}
0.070 \\
\end{tabular} & \begin{tabular}{|l|}
0.959 \\
\end{tabular} & 0.981 & 0.957 \\
\hline
\end{tabular}

The modified fitting indexes are improved obviously and reached the standard. Test results show that the structural model's goodness of fit that based on Bootstrap is acceptable, and the model can better fit the data. Therefore, we will test the research hypothesis proposed in this paper according to the path coefficient. The results are given in Table 6.

Table 6. Path Assumptions and Hypothesis Testing

\begin{tabular}{lllll}
\hline $\begin{array}{l}\text { Hypothesis Path hypothesis } \\
\text { Test results }\end{array}$ & & Path coefficient & p-value \\
\hline H1 & Network characteristics $\rightarrow$ Performance of KC & 0.22 & 0.053 & Support \\
H2 & Individual attributes $\rightarrow$ Performance of KC & 0.47 & 0.000 & Support \\
H3 & Team attributes $\rightarrow$ Performance of KC & 0.55 & 0.000 & Support \\
\hline
\end{tabular}

Note. Path coefficients are standardized coefficients.

When the significance level of the path coefficients is less than 0.05 , the hypothesis is true. When the significance level of path coefficient is less than 0.1 , it means that significance is weakly and hypothesis is partially supported. From the result we can see, path coefficient of network characteristics impact on performance of $\mathrm{KC}$ is 0.216 and its 
significance level is close to 0.05 , so the hypothesis 1 was established. Individual attributes has a significant positive effect on performance of $\mathrm{KC}(\mathrm{P}<0.001)$, hypothesis 2 is supported. Similarly, team attributes also has a significant positive impact on performance of $\mathrm{KC}$, hypothesis 3 was established.

\section{Research Conclusions and Implications}

\subsection{Research Conclusions}

At present, the virtual team is an important organizational form of knowledge creation in many fields, especially in IT service outsourcing industry. Creating virtual team, giving full play to the collective intelligence of their knowledge-based employees to create more new knowledge, new technologies and new products via creating efficient mechanism of collaborative working, is the target of many innovative enterprises. Structural characteristics of network are further verified in this paper through theoretical analysis and empirical research (network density and network centrality), and lifting the role of relation characteristics of network(connection strength) on efficieney ) of knowledge collaboration is also verified in this paper. The network density, network centrality and connection strength all have important effect on mproving performance of $\mathrm{KC}$ in virtual teams. And individual collaborative attention and capacity atl have positive influence on performance of $\mathrm{KC}$. It also found the influence relation of the atribute of the virtual team on performance of $\mathrm{KC}$, and verified the beneficial action of the team's collaborative atmosphere, collaborative culture and motivator on improving performance of $\mathrm{KC}$.

\subsection{Practical Implications}

Empirical conclusions will ptovide a theoretical basis and practical reference for promoting the performance of knowledge oolfaboration. Based on the research, this paper propose some advice to a virtual team

(1) Setting up knowledge collâboration work platform to provide team members with a good cooperative environment.

The enterprise should construct technology platform for collaborative work, provide their employees with internal unified platform for knowledge sharing and communication, business collaborationand collaborative operation, to reduce communication costs and to promote the smoothness of the knowledge circulation channels; and the enterprise should create collaborative knowledge base, on the one hand, to improve the efficiency of the team's knowledge sharing, and on the other hand, to provide sources and reserves of knowledge for the team's knowledge update and creation.

accessing.

Whether there are the structural characteristics of network density and network centrality, or the relation characteristic of connection strength, they all reflect that the closeness of the connection between the internal and external individuals of the team, playing a very important role in the effectiveness and efficiency of knowledge collaboration. Moreover, the appreciation of social capital is inseparable from breadth and depth of social network. Therefore, the strengthening of construction and maintenance of social network is an important mean to improve performance of $\mathrm{KC}$, appreciation of social capital and accumulation of knowledge capital. Especially for virtual teams without time-space limit, with fuzzy boundaries and flexible structure, effective and selforganized social network is a magic weapon to avoid drawbacks due to its flexibility.

(3) Creating a good collaborative culture and atmosphere. 
Virtual teams are strong support and organizational guarantee to carry out collaborative innovation. In virtual teams, it seems to be particularly important to establish confidence mechanism among team members due to characteristic including looseness, etc. of organization structure. Only if there is a good mutual trust relation among team members, the seamless joint of knowledge sharing can be completed, and the channel of knowledge flow can be smooth. Therefore, the construction of collaborative culture should be strengthened in virtual teams, and humanistic environment with mutual trust, being happy to share and convenient communication among team members is created from value orientation. Meanwhile, the team organizer should consciously encourage and promote the knowledge collaborative behavior within the team, and form the "Sharing among People", mutual understanding, mutual recognition and collaborative atmosphere which could main consistency on the tasks and targets gradually.

(4) Formulating constitutions and standardized system, adopting flexible motivation measures to stimulate team collaboration

Virtual team is the organizational carrier of knowledge dreation, in order to achieve knowledge creation, it needs smooth knowledge sharing and effective knowledge transfer in the first place, and all these need the subjective intrative of the relevant knowledge nodes within the virtual team and the efficient connection among them to achieve the knowledge transfer, digest, absorption and utilization effectively. Therefore, the organizer of the virtual team should adopt targeted an a flexible motivation measures directing at the personality of different members, formulate constitutions and standardize system to motivate the knowledge sharing intentions of the subject of knowledge creation and to improve its efficiency of knowledge collaboration. and give positive motivations to the activity and behavior helping to/promote the efiriency and performance of knowledge collaboration, so as to form the healthy motivation and feedback mechanism, to improve the efficiency of virtual team's knowtedge collaboration and to promote the creation and application of new knowledge ultimately.

\section{References}

[1] M. L. Maznevski and K. Thudoba, J. Bridging space over time: global virtual team dynamics and effectiveness. Organization science, vol. 5, no. 11, (2000).

[2] J. Lipnack and S. Jeffrey, Editor, Vitural teams: reaching across space, time and organizations with technology, Wiley, New York (1997)

[3] B.L.Kirkman, B. Rosen and C.B. Gibson, J. Five challenges to virtual team success: lessons from Sabre, Inc. The Academy of Management Executive.3, 16 (2002)

[4] I. Zigurs, I. Leadership in virtual teams: oxymoron or opportunity? Organizational Dynamics. 4, 31(2003)

[5] Chen Kah, J. Study on partner selection in virtual R\&D team based on fuzzy synthetic evaluation . Jounal of Henan Polytechnic University.1, 28 (2009)

[6] Li Yanyong and Zhu Shaoying, J. A study of current literature in virtual innovation teams in China basic on the I-P-O model. Science \& Technology Process and Policy. 4, 30 (2013)

[7] W. Karlenzig and J. Patrick, J. Tap into the power of knowledge collaboration. Customer Interaction Solutions. 11, 20 (2002)

[8] M. Mckelvey, H. Almb and M. RiccaboniI, J. Does co-location matter for formal knowledge collaboration in the Swedish Biotechnology-pharmaceutical Sector. Research Policy. 3, 32(2003)

[9] Tong Zehua, J. Knowledge Collaboration ( KC) and the relationship between KC and some related concepts. Library and Information Service. 8, 56 (2012)

[10] H.V. Leijen and W. R. J. Baets, Editors. A cognitive framework for reengineering knowledge intensive process. Proceedings of the 36th Annual Hawaii International Conference, (2003) Hawaii, USA.

[11] Chen Kunyu and Chen Kun-qiong, J. On company knowledge synergy. Information Science. 9, 20 (2002)

[12] Luo Min, Zhang Shengyi and LiuYongjun, J. The research on new and high technology enterprises know ledge governance performance. Science Research Management. 3, 31 (2010).

[13] Wang Youqing and Deng Ming-ran, J. Analysis about synergetic innovation performance of industry cluster based on angle of knowledge view. Science of Science and Management of S\&T. 4, (2009) 
[14] Du Baocang and Li Chaoming, J. Research on extension valuation on cooperative work performances of knowledge staff between organizations. Journal of Information.11, 28 (2009)

[15] Jan Zhaoquan, Liu Rong and Zhao Lizhu, J. The impact of network relationship, trust and knowledge sharing on technology innovation performance. R\&D Management. 2, 22 (2010)

[16] Xu Qiang and Zheng Xiaodan, J. Research on the relationship among the organizational collaboration, knowledge collaboration and innovation performance for parent-subsidiary companies. Science \& Technology Progress and Policy. 16, 27 (2010)

[17] Zhou Fang and Guo Yan, J. Research on the relationship among social capital, knowledge sharing and innovation performance for supply chain corporate. Research on Financial and Economic Issues. 12, 34 (2012)

[18] Shi Liping, Du Zewen and Liu Qiang, J. Study on the effect of transactive memory system on performance of knowledge team. Science \& Technology Progress and Policy. 8,30 (2013)

[19] Wu Shaobo and Gu Xin, J. Research on the knowledge synergy of knowledge chain's inter-firm cooperation. Science of Science and Management of S. \& T. 8, (2008)

[20] A.Seufert, G.V. Krogh and A.Bach, J. Towards knowledge networking. Journal of Knowledge Management, 3,3(1999)

[21] M.T. Hansen, J. The search-transfer problem: the role of weak ties in sharing knowledge across organization subunits. Administrative Science Quarterly. 1,44(1999)

[22] J. Singh, J. Collaborative networks as determinants of knowledge diffusion patterns Management Science. 5, 51 (2005)

[23] A. C. Inkpen, J. Tsang EWK. Social capital, networks and khowledge transfer. Academy of Management Review. 1,30 (2005)

[24] J. H. Y. Stephen and Y. L. C. Irene, J. A social network-based system for supporting interactive collaboration in knowledge sharing over peer-to-peer network. International Journal of HumanComputer Studies. 1,66 (2008)

[25] Zhu Yali, Xu Qing and Wu Xuhui, J. An empirical research on the effect of network density on interenterprise know ledge transfer: the mediating effeet of willingness of two parties. Studies in Science of Science. 3,29 (2011)

[26] Yanli Guo and Jianbin Chen, Editors A Case Study: Social network and knowledge sharing. Proceedings of the InternationaKConference on EAusiness and E-Government, (2010) May 14-16; Guangzhou, China.

[27] Shen Huimin, Ke Qing and Liu Gaoyong, ${ }^{\mathrm{J}}$. Building analytical model for knowledge collaboration community: an empirical stady. Journal of Intelligence. 2, 32 (2013)

[28] Chen Jianbin, Guo Yanliand Xu Kai-bo.J. Study on appraisement of knowledge collaboration based on knowledge capital appreciation. Science of Science and Management of S\&T. (2014)(Accepted)

[29] J. Nahapiet and S.Ghoshal, J. Social capital, intellectual capital, and the organizational advantage. Academy of Maragement Review 2, $32(1998)$

[30] Wang Xuedong Yi Ming and Zhan Wang-guo, J. The prospective of social network embeddedness on the knowledge Sharing in Yitual Teams. Information Science. 12, 27 (2009)

[31] M.A. McFadyen and A.A. Cannella, J. Social capital and knowledge creation: diminishing returns of the number and strength of exchange relationships. Academy of Management Journal. 5, 47 (2004)

[32] M. Fritsch and M. Kauffeld-Monz, J. The impact of network structure on knowledge transfer: an application of social network analysis in the context of regional innovation networks. The Annals of Regional Science. 1, 44 (2010)

[33] Chen lu, Zhao Zheng and Jing Runtian, J. An empirical study on impact of human relation network upon employee performance in virtual teams. Chinese Journal of Management. 9, 6 (2009)

[34] M. Reinholt, T. Pedersen and N. J. Foss, J. Why a central network position isn't enough: the role of motivation and ability for knowledge sharing in employee. Networks Academy of Management Journal.6,54 (2011)

[35] Yao Xiaotao and Xiu Youming, J. Management research and social network analysis. Modern Management Science. 6, (2008)

[36] R. Reagans and B. McEvily, J. Network structure and knowledge transfer: the effects of cohesion and range. Administrative Science Quarterly . 2, 48 (2003)

[37] B.Uzzi and R.Laneaster, J. Relational embeddedness and leaning: the case of bank loan managers and their clients. Management Science. 4, 49 (2003)

[38] Zhaoguo Xuan, Haoxiang Xia and Yanyan Du, J. Adjustment of knowledge-connection structure affects the performance of knowledge transfer. Expert Systems with Applications. 12, 38 (2011)

[39] Jente Yang, J. Knowledge management and innovativeness: The role of organizational climate and structure. Tourism Management. 2,39 (2008)

[40] K. E. Sveiby and R. Simons, J. Collaborative climate and effectiveness of knowledge work-an empirical study. Journal of Knowledge Management. 5, 6 (2002)

[41] S. P. López, J. M. M. Peón and C. J. V. Ordás , J. Managing knowledge: the link between culture and organizational learning. Journal of Knowledge Management. 6, 8 (2004)

[42] Li Guangsheng, Zhang Tao and Huang Jiewu, J. Research on the effect of collaborative culture and leadership roles on knowledge sharing. Science \& Technology Progress and Policy. 10, 26 (2009) 
[43] Luo Jiade, Editor, Social network analysis, Social Sciences Academic Press, China (2010)

[44] Zhu Yali, Empirical research on impacting factors of inter-enterprise knowledge transfer from the perspective of social network. Doctoral Dissertation of Zhejiang University, China (2009)

[45] M.Granovetter, J. The strength of weak ties. American Journal of Sociology. 6, 78 (1973)

[46] P.V.Marsden and K.E.Campbell, J. Measuring tie Ssrength. Social Forces.2, 63 (1984)

[47] Xie Xinlin, J. Influencing factors of knowledge work efficiency of team collaboration and its evaluation model. Doctoral Dissertation of Chongqing University, China(2010)

[48] Liu Jia-jia, Chen Tao \& Zhu Zhi-min, J. Research on the relationship between corporate social capital and knowledge sharing- knowledge acquisition as mediator. Science \& Technology Progress and Policy. 4, 30 (2013)

[49] I. Nonaka, J. A dynamic theory of organizational knowledge creation. Organization Science. 1, 5 (1994)

[50] Hau Kittai, Wen Zhonglin and Chen Zijuan, Editor, Structural equation model and its application, Educational Science Publishing House, Beijing, China (2004)

\section{Acknowledgements}

The authors gratefully acknowledge the Funding project of Beijing Philo\$ophy and Social Science Research Program (11JGB039), the Funding Project of outstanding talents from Beijing Organization Department (2012D005022000004)ånd the Funding project of research plan from the Education Committee of Beijing (SZ201311417001).

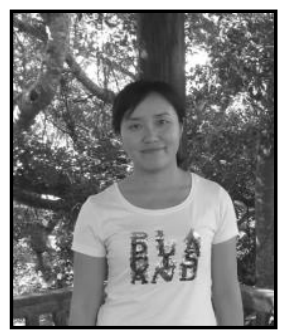

Shuli Gao

Authors

Doctor, Lecture of Department of E-business in Business College at Beijing Union niversity.

Address: A3 Yanjing Dong Li, Chaoyang District

Beijing, 100025, P.R. China

E-mail: shulr.gao@buu.edu.cn

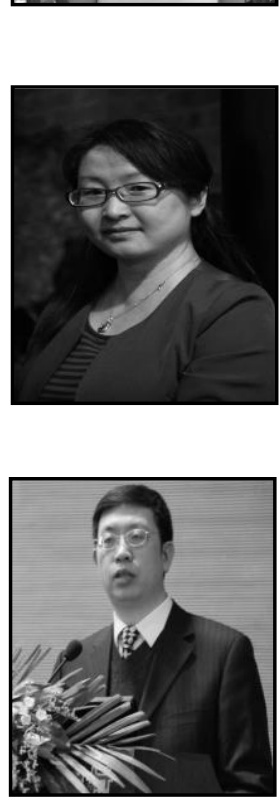

Yanli Guo

Doctor, Associate Professor of Department of E-business in Business College at Beijing Union University.

Address: A3 YanJing Dong Li, Chaoyang District,

Beijing, 100025, P.R.China.

E-mail: yanli.guo@buu.edu.cn

\section{Jianbin Chen}

Doctor, Professor of Department of E-business in Business College at Beijing Union University.

Address: A3 YanJing Dong Li, Chaoyang District,

Beijing, 100025, P.R.China

E-mail: jianbin.chen@buu.edu.cn 